\title{
A large pigmented lesion mimicking malignant melanoma
}

\author{
A. Brinca, V. Teixeira, M. Gonçalo and O. Tellechea \\ Dermatology Department, Coimbra University Hospital, Coimbra, Portugal
}

doi: $10.1111 / j .1365-2230.2012 .04464 . x$

\section{Clinical findings}

An 88-year-old woman was admitted with a huge bilateral pulmonary embolism and acute renal failure.

On physical examination, a well-defined irregularly pigmented plaque $20 \times 40 \mathrm{~mm}$ with black, brown and pink hues and an irregular border was noticed over the umbilicus (Fig. 1). A clinical diagnosis of malignant melanoma (MM) was suggested, and surgical excision planned. However, the patient's prognosis was poor; she was very dyspnoeic, with oxygen saturation levels of $<75 \%$, despite the treatment for pulmonary embolism, thus we chose to perform a biopsy to confirm the diagnosis, with the intention to proceed to treatment once the patient recovered from the embolism.

\section{Histopathological findings}

On histological examination, a full-thickness intraepidermal proliferation of atypical keratinocytes with overlying parakeratosis was seen, characterized by disordered maturation, mitosis at different levels of the epidermis, and focal dyskeratosis (Fig. 2). Strikingly, the atypical keratinocytes contained variable amounts of melanin granules. Melanophages also occurred focally in the underlying dermis.

What is your diagnosis?

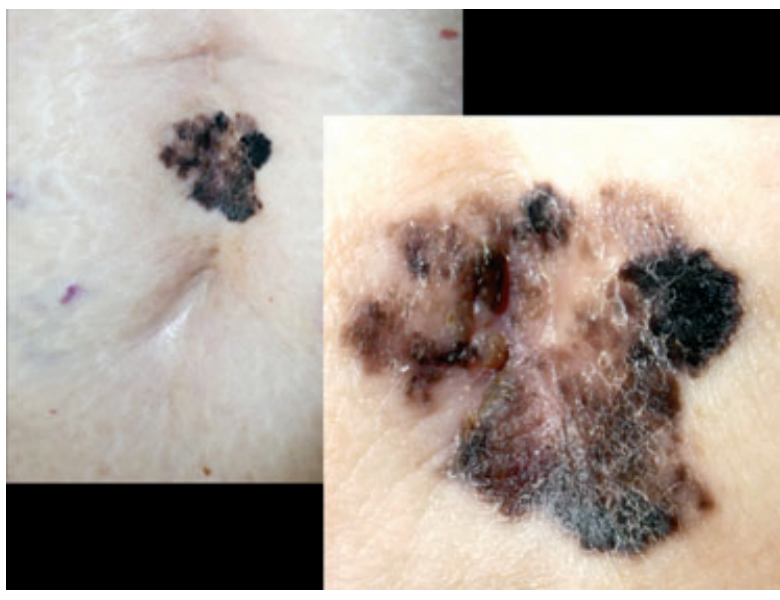

Figure 1 Pigmented Bowen disease over the umbilicus; (b) closeup view.

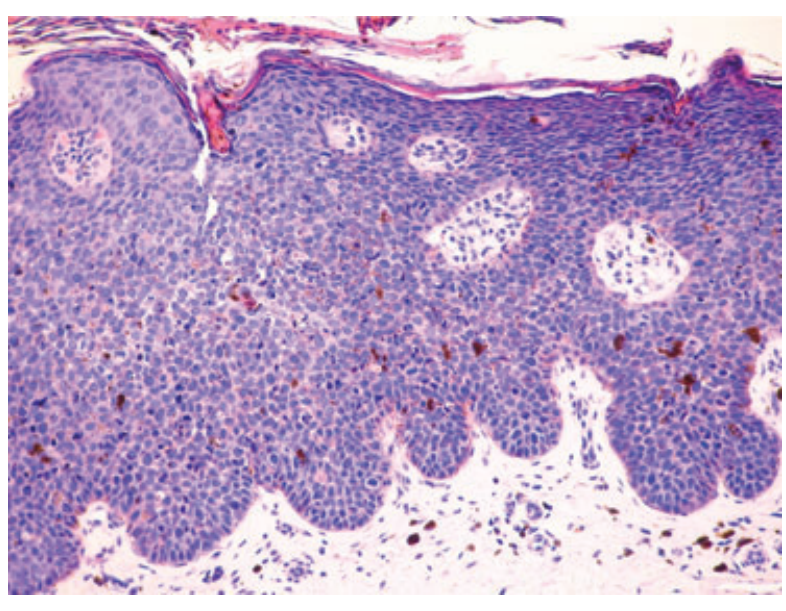

Figure 2 Intraepidermal proliferation of atypical keratinocytes, parakeratosis, disordered maturation, mitosis and focal dyskeratosis. Atypical keratinocytes contained variable amounts of melanin granules. Haematoxylin and eosin, original magnification $\times$ 200. 


\section{Diagnosis}

Pigmented Bowen disease (PBD).

\section{Discussion}

PBD is a rarely diagnosed cutaneous intraepidermal neoplasm that may be clinically difficult to distinguish from MM. It is a type of in situ squamous cell carcinoma (SCC), a malignant epithelial tumour, which presents as a slowly enlarging, pinkish, scaly plaque, and can affect both the skin and mucosa of older adults, especially women. In addition to exposure to ultraviolet radiation, other aetiological factors for SCC, have been suggested, including chemicals (e.g. arsenic), immunosuppression and human papillomavirus infection. Only about $5 \%$ of all cases of $\mathrm{BD}$ are pigmented ${ }^{1}$, and the condition is described more frequently in dark-skinned people. Unlike most types of in situ SCC, which occur mainly on sun-exposed areas, PBD is more likely to occur on sun-protected areas. In a recent retrospective analysis of 52 cases of PBD, Cameron et al. ${ }^{1}$ reported PBD as presenting most frequently as a flat or slightly raised, light-brown plaque, with varying degrees of scaling, occurring more often in men, on the limbs, trunk, head and neck. To our knowledge, there have only been two previous reports of PBD of the umbilicus. ${ }^{2,3}$

It is not fully understood why pigment deposition occurs. Some authors have suggested that neoplastic cells might produce specific cytokines that induce proliferation of melanocytes and production and transfer of melanin to the atypical keratinocytes, ${ }^{4}$ whereas others have suggested an association with solar lentigines and seborrhoeic keratoses. ${ }^{1,5}$ We did not find any histological evidence of these in our patient.

Clinically, PBD mimics other pigmented lesions such as seborrhoeic keratoses, pigmented actinic keratosis, solar lentigo, melanocytic naevi, pigmented basal cell carcinoma and MM, as in our patient. Various dermatoscopic features of PBD have been described but, according to Cameron, ${ }^{1}$ although a structureless pattern was the most common, the pattern of a linear arrangement of brown and/or grey dots and/or coiled vessels was more specific.

The presence of pigment does not change the prognosis or treatment of $\mathrm{BD}$, so treatment options include those indicated for nonpigmented BD: surgery, cryotherapy, ${ }^{6}$ laser ablation, topical imiquimod or 5-fluorouracil, curettage, electrosurgery and photodynamic therapy. ${ }^{3}$ PBD generally has a good prognosis; unfortunately, in this case, the patient's general condition was poor. We treated her with optimum anticoagulant therapy for the embolism, but her condition quickly deteriorated and she died 3 days later.

In conclusion, we report a patient presenting with a lesion that clinically was suggestive of MM. Unexpectedly, however, the histopathological finding identified the lesion as being a PBD. In general, the good prognosis of PBD permits a more conservative and cost-effective therapeutic approach of the lesion.

\section{Learning points}

- Only about $5 \%$ of all cases of BD are pigmented.

- Clinically, PBD presents most often as a flat or slightly raised, light-brown plaque, with varying degrees of scaling, occurring more often in men, on the limbs, trunk, head and neck.

- PBD should be included in the differential diagnosis of pigmented lesions such as MM.

\section{References}

1 Cameron A, Rosendahl C, Tschandl P et al. Dermatoscopy of pigmented Bowen's disease. J Am Acad Dermatol 2010; 62: 597-604.

2 Papageorgiou PP, Koumarianou AA, Chu AC. Pigmented Bowen's disease. Br J Dermatol 1998; 138: 515-18.

3 Lee JW, Hur J, Yeo KY et al. A case of pigmented Bowen's disease. Ann Dermatol 2009; 21: 197-9.

4 Krishnan R, Lewis A, Orengo IF et al. Pigmented Bowen's disease (squamous cell carcinoma in situ): a mimic of malignant melanoma. Dermatol Surg 2001; 27: 673-4.

5 Marschall SF, Ronan SG, Massa MC. Pigmented Bowen's disease arising from pigmented seborrheic keratoses. J Am Acad Dermatol 1990; 23: 440-4.

6 Mortimer PS, Sonnex TS, Dawber RP. Cryotherapy for multicentric pigmented Bowen's disease. Clin Exp Dermatol 1983; 8: 319-22. 